\title{
Bagatellfälle sind keine Bagatellen
}

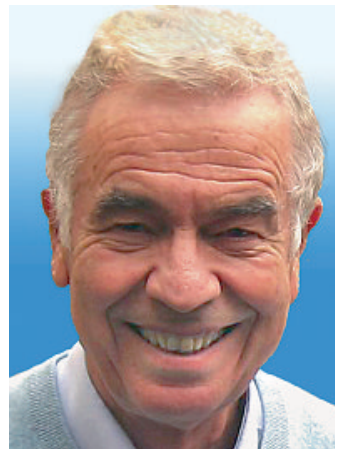

Hans Stalder

Aus einer im Februar dieses Jahres im Swiss Medical Weekly SMW [1] veröffentlichten Studie geht hervor, dass jede siebte Person im Kanton Genf aus wirtschaftlichen Gründen auf medizinische Leistungen verzichtet. Dabei handelt es sich vor allem um zahnärztliche Versorgung, aber auch um Arztkonsultationen, Medikation, Apparate, und selbst auf operative Eingriffe wird verzichtet. Das Problem betrifft besonders Personen in den unteren Einkommensklassen, aber auch Patienten mit kardiovaskulären Komorbiditäten, mit anderen Worten diejenigen, die aufgrund ihres hohen Erkrankungs- und Todesrisikos am dringendsten der ärztlichen Betreuung bedürften.

Bereits eine frühere Studie [2] hatte zutage gebracht, dass 7,5\% der hospitalisierten betagten Patienten zuvor aus finanziellen Gründen auf einen Arztbesuch verzichtet hatten.

Mit anderen Worten: Es finden weniger Konsultationen statt. Sollten wir nicht froh darüber sein? Zeigen uns diese Studien nicht, dass das schweizerische System mit Franchise und Selbstbeteiligung an den Gesundheitskosten funktioniert? Handelt es sich hier nicht um eine politische Zielsetzung, mit der «Bagatellfälle» vermieden werden sollen?

\section{Jeder Siebte verzichtet im Kanton Genf aus Kosten- gründen auf medizinische Versorgung.}

* Prof. Dr. med. Hans Stalder, Facharzt FMH für Innere Medizin FMH, Redaktionsmitglied, ist ehemaliger Direktor der Policlinique de Médecine und des Départements de Médecine communautaire des Hôpitaux Universitaires de Genève.
Der Gedanke, die Gesundheitskosten durch eine finanzielle Beteiligung der Patienten an den Konsultationen zu senken, stützt sich auf eine grosse Studie der RAND-Corporation aus den 1970er Jahren [3]. Das Prinzip erscheint wirtschaftlich und psychologisch sinnvoll: Wenn ich etwas gratis bekomme, neige ich dazu, es auszunutzen. Dies wird häufig mit dem englischen Begriff moral hazard, also als subjektives oder moralisches Risiko bezeichnet. Oder anders ausgedrückt: Wenn ich wirklich krank bin, gehe ich zum Arzt, selbst wenn ich es bezahlen muss.

Aber das Gesundheitssystem ist komplex, und lineare und auf den ersten Blick logische Eingriffe bringen häufig unerwartete Effekte.

Wenn man Allgemeinmediziner befragt, ob sie mit Bagatellfällen überlastet sind, so verneinen sie dies im Allgemeinen und beklagen sich höchstens über die von den Arbeitgebern verlangten Zeugnisse für Arbeitsunfähigkeit. Viele Bagatellfälle erweisen sich erst nach dem Arztbesuch tatsächlich als solche. Zudem erlaubt jede Konsultation präventive Unter- suchungen (Blutdruckmessung, Gespräch über Rauchen während einer Erkältung, über Alkohol bei Oberbauchbeschwerden usw.). Und schliesslich haben sogenannte «Bagatellen» - Erkältungen und Rückenschmerzen - hohe wirtschaftliche Folgen, denn sie sind die häufigste Ursache für Arbeitsunfähigkeit.

Im Übrigen haben verschiedene Studien, wie auch die oben erwähnte [1] gezeigt, dass häufig zuallererst auf lebenswichtige Medikamente verzichtet wird, wenn eine Kostenbeteiligung gefordert ist. Am meisten beunruhigen aber Ergebnisse aus detaillierteren und im Anschluss an die RAND-Studie durchgeführten Untersuchungen, denen zufolge die Einführung oder Erhöhung einer finanziellen Beteiligung einerseits zwar die Zahl der ambulanten Konsultationen senkt, andererseits aber die Zahl und Dauer der Hospitalisationen bei bestimmten Risikogruppen wie betagten Personen mit chronischen Erkrankungen oder bei sozial schwachen Personen mit geringem Bildungsniveau erhöht (Literatur hierzu s. [4]). Statt zu sinken, steigen die Gesundheitskosten in diesen Bevölkerungsgruppen also sogar an.

Diejenigen, die mithilfe einer Kostenbeteiligung der Patienten die «Bagatellfälle» aus unseren ambulanten Praxen verschwinden lassen wollen, müssten daraus eigentlich einige unliebsame Schlussfolgerungen ziehen: Zwar werden die Kosten bei jenen gesenkt, die kaum ärztliche Versorgung benötigen, nämlich bei jungen, reichen und gesunden Personen hier sind nur die Präventionsmassnahmen betroffen. Auf der anderen Seite treibt dieselbe Kostenbeteiligung die Gesundheitskosten bei den älteren Personen mit chronischen Krankheiten aber in die Höhe. Und dies ist ja gerade die Gruppe, die schon jetzt das Gesundheitssystem am teuersten zu stehen kommt.

Hans Stalder*

\section{Literatur}

1 Wolff H, Gaspoz JM, Guessous I. Health care renunciation for economic reasons in Switzerland. Swiss Med Wkly. 2011;141:w13165.

2 Coutaz M, Morisod J. Quelle perception les personnes âgées ont-elles des coûts médicaux qu'elles induisent? Rev Med Suisse. 2007;3:1912-5.

3 Newhouse JP, Manning WG, Morris CN, Orr LL, Duan N, Keeler EB, Leibowitz A, Marquis KH, Marquis MS, Phelps CE, Brook RH. Some interim results from a controlled trial of cost sharing in health insurance. N Engl J Med. 1981;305:1501-7.

4 Trivedi AN, Moloo H, Mor V. Increased ambulatory care copayments and hospitalizations among the elderly. N Engl J Med. 2010;362:320-8. 